\title{
Exponential and Power-Law Contact Distributions Represent Different Atmospheric Conditions
}

\author{
A. M. Reynolds
}

Rothamsted Research, Harpenden, Hertfordshire, AL5 2JQ, UK.

Accepted for publication 12 July 2011.

\begin{abstract}
Reynolds, A. M. 2011. Exponential and power-law contact distributions represent different atmospheric conditions. Phytopathology 101:14651470.

It is well known that the dynamics of plant disease epidemics are very sensitive to the functional form of the contact distribution-the probability distribution function for the distance of viable fungal spore movement until deposition. Epidemics can take the form of a constant-velocity travelling wave when the contact distribution is exponentially bounded. Fat-tailed contact distributions, on the other hand, lead to epidemic spreads that accelerate over time. Some empirical data for contact distri-

and inverse power laws are not competing candidate forms of the contact distribution but are instead representative of different atmospheric conditions. Contact distributions for atmospheric boundary-layers with stabilities ranging from strongly convective (a hot windless day time scenario) to stable stratification (a cold windy night time scenario) but without precipitation events are calculated using well-established state-of-the-art Lagrangian stochastic (particle tracking) dispersal models. Contact distributions are found to be well represented by exponentials for strongly convective conditions; a $-3 / 2$ inverse power law for convective boundarylayers with wind shear; and by a $-2 / 3$ inverse power law for stably stratified conditions.
\end{abstract} butions can be well represented by negative exponentials while other data are better represented by fat-tailed inverse power laws. Here we present data from numerical simulations that suggest that negative exponentials
Additional keywords: atmospheric dispersion, dispersal patterns, Lagrangian stochastic modeling, plant disease epidemics.
Plant disease epidemics develop inside plant canopies through the repeated dispersal of pathogens to healthy host tissue. Without dispersal, an epidemic would simply burn out. Fungal spores are the dispersal agent for many plant diseases, and atmospheric dispersal is the predominant mechanism for transporting the spores of the pathogens to healthy plant tissue (2). Accurate prediction of the spread of plant disease by aerially dispersed pathogens therefore requires knowledge of the number of pathogenic spores deposited onto susceptible plant tissues. Policy is often concerned with managing disease outbreaks caused by spores from unknown sources, and over spatiotemporal scales outside the practical capability of detailed models that account for local topography and weather conditions (36). In such situations, dispersal is often characterized in terms of a contact distributionthe probability distribution function for the distance of fungal spore movement until deposition. Contact distributions for fungal spores, as well as for pollens and seeds have commonly been represented by negative exponentials and by inverse power-laws which have 'fat' tails that cannot be bounded by exponentials $(1,5,10,11,15,21,24,36,43)$. Combinations of exponentials and inverse power-laws have also been suggested (6).

The functional form of the contact distribution determines the dynamics of an epidemic and so is of considerable practical interest. Mollison (29) showed that an epidemic can take the form of a travelling wave only when the contact distribution is exponentially bounded. Models with inverse power-law contact distributions, on the other hand, predict that the spread of an epidemic accelerates over time as a dispersive'wave $(8,13,19,22)$. This unbounded acceleration cannot apply indefinitely. Nonetheless, the

Corresponding author: A. M. Reynolds

E-mail address: andy.reynolds@ rothamsted.ac.uk

doi:10.1094/PHYTO-01-11-0001

(c) 2011 The American Phytopathological Society accelerating spread can dominate over ecological time if the contact distribution is sufficiently fat-tailed for long-distance dispersal (19). Related studies of focal expansion with power-law contact distributions have shown that dispersal produces a patchy, fractal-like, spatial population structure very different from the structure of a homogeneous front found with exponential contact distributions $(7,25,35,51)$. This clustering could result in new foci of infection beyond the original source, and clustering of disease around the new infections.

Attempts to characterize accurately the contact distribution thus resonate with the wider debate on the spatiotemporal dynamics of epidemic expansion of plant diseases from foci; a subject which Zadoks (52) identified as being one of the key controversies in plant disease epidemiology in the 20th century.

Recent attempts to predict contact distributions for aerially dispersed spores can be traced back to Ferrandino (13). Ferrandino (13) suggested rightly that because more and larger atmospheric eddies participate in turbulent transport at increasingly large spatial scales, atmospheric dispersal may not be characterized by an exponential contact distributions but could instead be characterized by fat-tailed contact distributions. Katul et al. (21) subsequently predicted more concretely that the contact distribution as a $-3 / 2$ inverse power-law tail. Katul et al. (21) made use of Lagrangian stochastic (LS) (particle-tracking) models. Over the past few years, LS models have frequently been advocated as a way to produce realistic trajectories of biological aerosols $(2,4,20,23,31,32)$. Other studies have shown that they describe accurately tracer-particle dispersion in a diverse range of complex turbulent flows including atmospheric boundary-layers, ocean gyres, and those within and above plant canopies $(16,33,34,44)$. Nonetheless, to obtain analytical results, Katul et al. (21) had to assume that atmospheric turbulence is vertically homogeneous. This seemingly innocuous assumption has a significant but overlooked consequence-it inevitably leads to contact distributions having $-3 / 2$ inverse power-law tail. This is because LS models 
for the simulation of particle trajectories in homogeneous turbulence reduce to homogeneous diffusion equations in the long-time limit (41) which by virtue of the Sparre Andersen Theorem $(37,38)$ produce time-of-flight distributions with a universal $-3 / 2$ power-law tail. In the presence of uniform mean flow, these time-of-flight distributions translate into contact distributions with $-3 / 2$ inverse power-law tails. Thus, it is not surprising to find that Stockmarr et al. (39) obtained results directly analogous to those of Katul et al. (21) by presupposing that atmospheric dispersal can be faithfully modeled using a homogeneous diffusion equation.

The vertical structure of turbulence can, however, impact significantly on the functional form of the contact distribution, as predicted by diffusion models. The results of numerical simulations reveal that diffusion models produce contact distributions that decay more slowly than a $-3 / 2$ inverse power-law when diffusivity increases linearly with height (data not shown). Contact distributions with exponential tails are produced when diffusivity increases exponentially with height or when diffusive transport has an upper ceiling (data not shown). A potentially more serious problem with the analyses of Katul et al. (21) and Stockmarr et al. (39) is that the employment of diffusion equations is only justifiable for weakly energetic, nearly homogeneous turbulent flows (40).

Further progress in predicting and in understanding contact distributions can be made by taking explicit account of the vertical structure of turbulence and by taking better account of turbulent transport processes. Here this challenge is addressed by fully utilizing LS models (34). These models are used to calculate contact distributions for atmospheric boundary-layers with stabilities ranging from strongly convective to stably stratification but without precipitation events. Strong convection occurs on hot days when air is heated from below by the ground. The heated air becomes buoyant and rises causing additional or even major turbulence. Turbulent dispersion is then strongly influenced by the relative probability of occurrence of convective updrafts and downdrafts. Updrafts have large vertical velocities but occupy less horizontal area while downdrafts occupy large area but have smaller velocities (26). Hence, the probability density function (pdf) of vertical velocities at a particular height is positively skewed (with skewness $\sim O(1)$ ), i.e., the mode is negative and not equal to the mean. The atmosphere can become stably stratified on cold nights when air is cooled from below by a ground. Vertical movement then becomes difficult, and small vertical disturbances in the air flow dampen out and disappear.

In the next section it is shown how epidemic dynamics can be deduced directly from the functional form of the contact distribution using general mathematical considerations that appear not to have surfaced in the literature on plant disease epidemics. LS models are then used to assess the dependency of the contact distribution on atmospheric conditions. The results of these numerical simulations suggest that negative exponentials and inverse power-laws are not competing candidate forms of the contact distribution but are instead representative of different atmospheric conditions.

\section{THEORY AND APPROACHES}

Contact distributions and epidemic dynamics. When the density of healthy host plants is not limiting, spatiotemporal disease dynamics depend only on the contact distribution and are governed by

$$
p_{n}(x)=R_{0} \int_{-\infty}^{\infty} p_{n-1}(s) P(x-s) d s
$$

where $p_{n}(x)$ is the disease density at position $x$ after $n$ generations (cycles), $R_{0}$ is the basic reproduction number, and $P(x)$ is the contact distribution.
If the second-moment of the contact distribution is well-defined (i.e., if

$$
\left.\int_{0}^{\infty} x^{2} P(x) d x \neq \infty\right)
$$

as is the case when the contact distribution is exponentially bounded, then solutions to equation 1 for the disease-density profiles will tend to Gaussian distributions

$$
p_{n}(x)=\frac{R_{0}^{n}}{\sqrt{2 \pi n} \sigma} \exp \left(-\frac{x^{2}}{2 n \sigma^{2}}\right)
$$

by virtue of the central limit theorem. And consequently, the average rate of epidemic expansion tends to a constant $x^{\prime} / n \rightarrow \sigma \sqrt{2 \ln R_{0}}$, where $x^{\prime}$ is the position at which the disease density $p_{n}\left(x^{\prime}\right)=p^{\prime}$, a marker for significant disease. This constant rate of expansion is indicative of travelling wave epidemics. If the contact distribution has a power-law tail $P(x) \propto x^{-\mu}$ with $1<\mu<3$, then its second-moment is a divergent quantity and the solution to equation 1 for the disease-density profile will tend to a Lévy stable distribution by virtue of a generalized central limit theorem (17). These Lévy stable distributions have inverse powerlaw tails and satisfy the scaling relation

$$
p_{n}(x)=\frac{R_{0}^{n}}{n^{1 /(\mu-1)}} p_{1}\left(\frac{x}{n^{1 /(\mu-1)}}\right)
$$

The spatiotemporal dynamics of these epidemics are therefore manifestly "self-similar" since the disease-density profile retains its shape as the epidemic develops. Furthermore, because

$$
p_{n}(x) \rightarrow \frac{R_{0}^{n}}{n^{1 /(\mu-1)}}\left(\frac{x}{n^{1 /(\mu-1)}}\right)^{-\mu} \text { as } x \rightarrow \infty
$$

the position at which the disease density $p_{n}\left(x^{\prime}\right)=p^{\prime}$ is given by

$$
x^{\prime}=\left(\frac{n}{p^{\prime}}\right)^{\frac{1}{\mu}} R_{0}^{n / \mu} \equiv\left(\frac{n}{p^{\prime}}\right)^{\frac{1}{\mu}} \exp \left(\frac{n}{\mu} \ln R_{0}\right)
$$

The average rate of epidemic expansion, $x^{\prime} / n$, therefore increases exponentially with generation. This is indicative of dispersive wave epidemics.

LS simulations. Each simulated spore is released from a point source with a velocity drawn at random from a distribution that is consistent with prescribed meteorological data for turbulent air velocity statistics at that location. Its subsequent trajectory through the atmospheric boundary-layer is then simulated by numerically integrating a LS model. LS models for the simulation of particle trajectories in atmospheric turbulence take the general form

$$
d u_{i}=a_{i}(\mathbf{x}, \mathbf{u}, t) d t+\sqrt{\mathrm{d} \mathbf{x}=\mathbf{u} d t}\left(\mathrm{C}_{0} \varepsilon \xi_{i}\right.
$$

where bold quantities denote vectors, the subscripts denote Cartesian components, $\mathbf{x}$ and $\mathbf{u}$ are the position and velocity the particle at time $t, C_{0}=3$ is a universal constant, $\varepsilon$ is the mean rate of dissipation of turbulent kinetic energy divided by the density of air, and $d \xi_{i}$ are random Gaussian variants with mean zero and variance $d t(41)$. The function $a_{i}(\mathrm{x}, \mathrm{u}, \mathrm{t})$ is chosen so that the pdf of the velocities of simulated particles passing through any volume matches the Eulerian pdf of the measured velocities within that volume. This natural condition, known as the "wellmixed condition," provides the most rigorously correct theoretical framework for the formulation of LS models, and is appropriate when particle trajectories are largely unaffected by particle inertia (41). It ensures that simulated particle velocities are consistent with measured turbulent velocity statistics that are used as model inputs. 
Here particle dispersal in atmospheric boundary-layers over flat terrain was simulated using the two-dimensional (stream-wise and vertical movements with velocity components $u$ and $w$ ) LS model devised by Rotach et al. (34) which fulfils the well-mixed condition for stabilities ranging from neutral to convective conditions. In this model the pdf of particle velocities is the weighted sum of a neutral pdf ( $u$ and $w$, are jointly Gaussian) and a convective $\operatorname{pdf}$ ( $w$ is skewed, $u$ and $w$ are uncorrelated). The convective pdf is modeled as the weighted sum of two Gaussian distributions that are representative of velocities within the updrafts and downdrafts. The weights are the possibilities of occurrence of updrafts and downdrafts. They can also be considered as the areas occupied by updrafts and downdrafts. The transition function varies continuously with stability. The model is parameterized using data drawn from field studies and from laboratoryscale experiments. A complete description of this model and its parameterization can be found in Rotach et al. (34). For purely convective conditions, the model reduces to that described in Luhar and Britter (26), which is in good agreement with laboratory measurements of Willis and Deardorff (48-50). Model predictions are also in good agreement with those of Mason (27) who used large-eddy simulations to assess dispersion characteristics for a range of atmospheric conditions (18). Crosswind movements were simulated using a separate one-dimensional LS model for homogenous turbulence (41). Required crosswind turbulent statistics were approximated by stream-wise turbulent statistics because more accurate parameterizations are not available. This is not overly restrictive as predictions for the functional form of the contact distribution do not change when the crosswind turbulent statistics were approximated by $1 / 2$ of stream-wise turbulent statistics. Additionally, simulations were carried out for stable conditions using a LS model for one-dimensional turbulence with Gaussian velocity statistics (41) and utilizing velocity statistics drawn from the simulation data of Weil et al. (46). The numerical simulations of Weil et al. (46) reproduce several characteristic features of stable nocturnal boundary-layers including the presence of low-level jets and realistic turbulence profiles. The LS model was used to simulate the vertical motion of passive particles that are advected downwind by the mean flow. This is appropriate because stream-wise diffusion plays a minor role in dispersion under stable conditions (12).

For the most part, the trajectory of a spore through the atmosphere will be largely unaffected by inertia. An important exception to this occurs very close to flow boundaries, i.e., very close to the ground and close to plant surfaces. Close to a flow boundary, spore trajectories can become disentangled from the very weak turbulence there and then be carried by their virtue of their inertia to the boundary where they can subsequently be deposited. Tracer particles, on the other hand, can never reach a flow boundary. For this reason, LS simulations of tracer-particle trajectories are usually implemented using reflective boundary conditions, as it is computationally prohibitive to resolve all near-boundary smallscale turbulent motions (42). Here to facilitate the simulation of spore dispersal, LS simulations were implemented with nonreflective absorbing boundary conditions. This amounts to assuming that spores are deposited when they first reenter the plant canopy.

For each of the atmospheric stabilities considered, contact distributions were calculated by simulating the trajectories of $10^{6}$ particles from their release from a point source at height $0.001 \mathrm{H}$ until they first reached the ground. The height, $\mathrm{H}$, of a stable boundary-layers is typically between 200 to $300 \mathrm{~m}$, while strongly convective boundary-layers can extend upwards several kilometers. Model predictions for the asymptotic form of the contact distribution do not change when the source height is increased or decrease by a factor of 10 . The distribution of distances $\mathrm{x}$, travelled downwind by the particles defines the contact distribution. Analogous results (not shown) were obtained for the distri- bution of net distances travelled $\sqrt{x^{2}+y^{2}}$ by spores where $y$ is the distance travelled in the crosswind direction. Turbulence within and just above the plant canopies is not accounted for explicitly because it is assumed that long-distance spore dispersal takes place within the atmospheric boundary-layer after spores have been carried upwards out of the plant canopy by turbulent gusts (ejections) (14). Spore mortality due to desiccation and exposure to ultraviolet radiation was not accounted for. These factors are most significant under strongly convective conditions and favor an exponential contact distribution, as do precipitation events.

For illustrative purposes binned simulation data for contact distributions are presented on log-log scales. A straight-line on such a plot is indicative of power-law scaling but the gradient of a straight-line does not necessarily provide a reliable indicator of the power-law exponent (47). Perhaps the most reliable indicator of power-law scaling is the Akaike weight (47). The Akaike weight for the power-law can be considered as the weight of evidence in favor of the power-law being the better model, out of the models considered, of the simulation data, i.e., the Akaike weight for a power-law can vary from 0 (no support) to 1 (complete support). Here truncated power-law distributions $\left(P_{1}(l) \propto l^{-\mu}\right.$ for $10 H \geq l \geq 0.1 H$ otherwise $\left.P_{1}(l)=0\right)$ are compared with truncated negative exponential distributions $\left(P_{2}(l) \propto \exp (-\lambda l)\right.$ for $10 H \geq l \geq 0.1 H$ otherwise $\left.P_{2}(l)=0\right)$. Following White et al. (47), power-law exponents are estimated using maximum likelihood methods.

\section{RESULTS}

The contact distribution is predicted to be exponential for strongly convective boundary-layers (Fig. 1A). The Akaike weight for a power-law is 0.0 .

For a wide range of daytime conditions the contact distribution is predicted to have a universal inverse power-law tail. The Akaike weight for a power-law is 1.0 and the maximum likelihood estimate for the power-law exponent is 1.5 (Fig. 1B). The tail does not change when the atmospheric conditions change over the time of spore release. It arises, for example, when the surface heat flux remains constant (approximately $30 \mathrm{Jm}^{-2} \mathrm{~s}^{-1}$ ) whilst the mean wind speed ranges between $0 \mathrm{~ms}^{-1}$ and about $13 \mathrm{~ms}^{-1}$ during the day, and arises when the friction velocity (i.e., a measure of the near ground-level wind speed) remains constant whilst the daily surface heat fluxes ranges between 0 and $240 \mathrm{Jm}^{-2} \mathrm{~s}^{-1}$. Exponential contact distributions do, however, arise if spores are released in strongly convective conditions, become distributed throughout the boundary-layer, and then strong convection gives way to windy conditions.

Under stable conditions, the contact distribution is predicted to have a near $-2 / 3$ inverse power-law tail (Fig. 1C). The Akaike weight for a power-law is 1.00 and the maximum likelihood estimate for $\mu=0.7$. This scaling extends at least to distances $1000 \mathrm{H}$ downwind of the source, where $\mathrm{H}$ is the height of the boundary-layer but cannot extend to arbitrarily large distances because normalization (i.e., the necessary condition that probabilities sum to unity) requires that $\mu>1$. It is computationally prohibitive to simulate dispersion to much greater distances. Diffusion models for weakly turbulent stably-stratified flows predict that contact distributions have near -1 inverse power-law tails (data not shown).

In accordance with previous studies $(7,25,35,51)$ predicted dispersal patterns under strongly convective conditions (i.e., for exponential contact distributions) are localized around the source and a have well defined focus (Fig. 2A) while dispersal patterns on windy days are patchy and far greater numbers of spores are predicted to be deposited far from the source (Fig. 2B). The asymmetry seen in Figure $2 \mathrm{~A}$ arises because unlike crosswind movements, upwind and downwind movements are correlated with vertical movements. 


\section{DISCUSSION}

A Convective

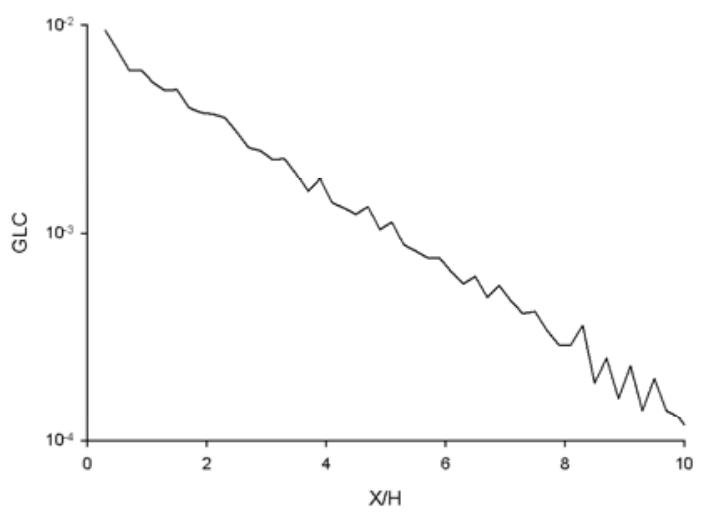

B Convective boundary-layer with wind shear

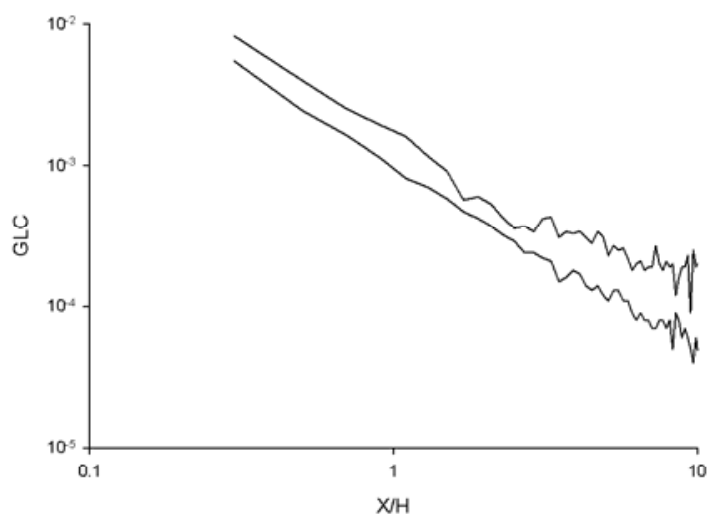

C Stable

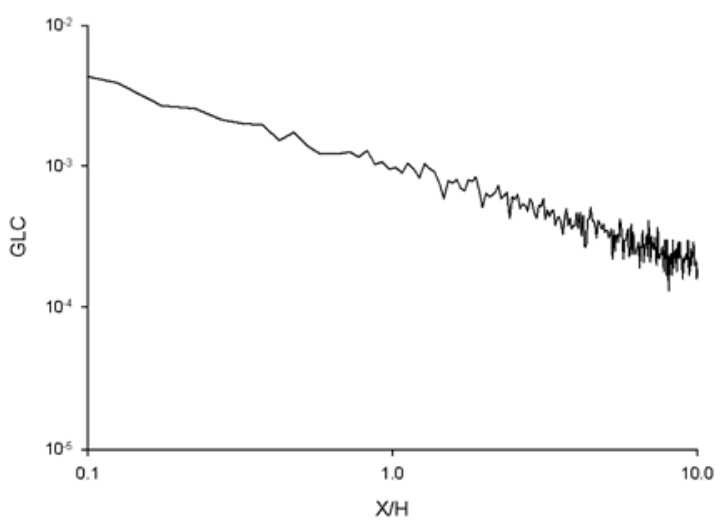

Fig. 1. Predicted contact distributions, i.e., the normalized ground-level concentrations (GLC) of fungal spores at distances, $x$, downwind of a point source of height $0.001 \mathrm{H}$. Predictions are shown for $\mathbf{A}$, an almost purely convective boundary $\left(u_{*}=0.01 \mathrm{~ms}^{-1}, w_{*}=2.0 \mathrm{~ms}^{-1}\right)$; $\mathbf{B}$, a convective boundary-layers with modest wind shear with $u_{*}=0.4 \mathrm{~ms}^{-1}, w_{*}=0.7 \mathrm{~ms}^{-1}$ (top curve) and with $u_{*}=0.8 \mathrm{~ms}^{-1}, w_{*}=0.5 \mathrm{~ms}^{-1}$ (bottom curve) and; C, a stably stratified boundary-layer with an Ekman spiral and low level jet $\left(u_{*}=0.28 \mathrm{~ms}^{-1}\right.$ ). Here $u_{*}$ is the friction velocity (square root of the surface stress divided by the density of the air) and $w_{*}=\left(-h_{*} H\right)^{1 / 3}$ is the convective velocity where $h_{*}$ is surface heat flux. $w_{*}=1 \mathrm{~ms}^{-1}$ corresponds to a small surface heat flux, $h_{*}$, of approximately $30 \mathrm{Jm}^{-2} \mathrm{~s}^{-1}$ (buoyancy flux $-10^{3} \mathrm{~m}^{2} \mathrm{~s}^{-1}$ ). $u_{*}=1 \mathrm{~ms}^{-1}$ corresponds to a mean wind speed (average over the boundary-layer) of approximately $12.9 \mathrm{~ms}^{-1}$.
The aerial dispersal of spores was simulated using LS models for atmospheric conditions ranging from strongly convective to stably stratification. In the long-time limit these models reduce to diffusion equations when inhomogeneity is weak, i.e., when the timescale on which turbulence as viewed by a particle changes due to inhomogeneity is much longer than the timescale over which particle velocities remain significantly correlated. For stronger inhomogeneity it is not clear whether LS models can or should reduce to diffusion equations (41).

Over an extended range of daytime conditions, contact distributions are predicted to have $-3 / 2$ inverse power-law tail. This prediction stems from atmospheric dispersal processes rather than from the employment of homogeneous diffusion equations, and so is different in origin from the $-3 / 2$ scaling uncovered by Katul et al. (21) and Stockmarr et al. (39). The $-3 / 2$ inverse power-law tail predicted by the LS models is supported by a raft of empirical studies of pollen and seed dispersal $(1,5,11,21,36)$. Departures from $-3 / 2$ scaling arise under strongly convectively conditions (hot windless or nearly windless days) for which the contact distribution is predicted to be exponential, and under
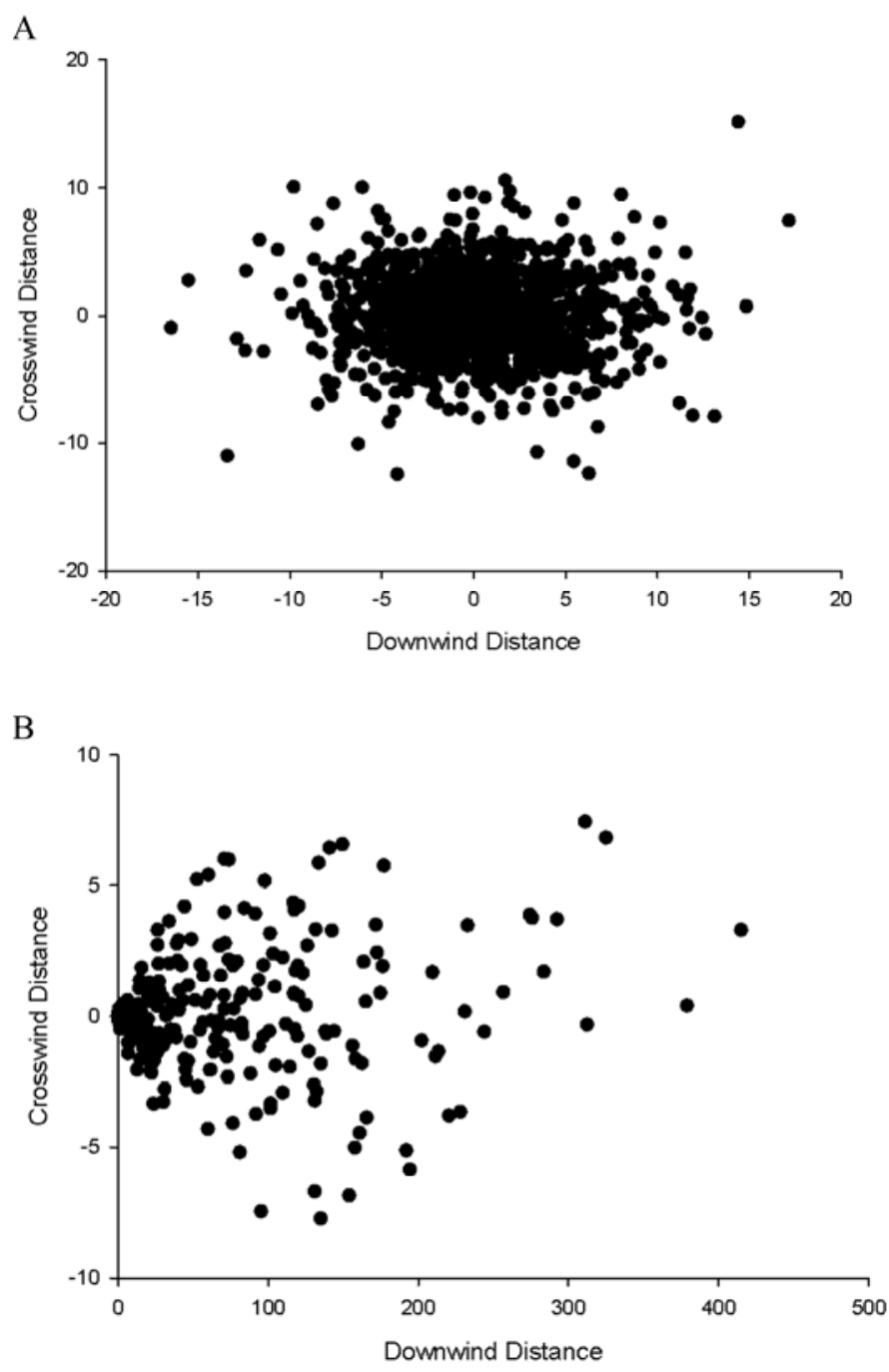

Fig. 2. Predicted dispersal patterns produced by simulating the trajectories of 10,000 fungal spores from their release from a point source of height $0.001 \mathrm{H}$ located at the origin to their deposition at ground level. The deposition sites are marked $(\bullet)$. Distances are in terms of the boundary-layer height, $\mathrm{H}$. Predictions are shown for $\mathbf{A}$, an almost purely convective boundary $\left(u_{*}=0.01 \mathrm{~ms}^{-1}, w_{*}=2.0 \mathrm{~ms}^{-1}\right)$ and $\mathbf{B}$, a convective boundary-layer with modest wind shear $\left(u_{*}=0.8 \mathrm{~ms}^{-1}, w_{*}=0.5 \mathrm{~ms}^{-1}\right)$. 
stable conditions (cold nights) for which the contact distribution is predicted to have $-2 / 3$ power-law tail. The latter prediction is in accordance with classic field data from Project Prairie Grass (3) and with data from the numerical simulations (45). Despite its age, Project Prairie Grass remains the most extensive and the most successful short-range field dispersion experiment. In these experiments, $\mathrm{SO}_{2}$ was released at a height of $0.5 \mathrm{~m}$ at a site covered with grass. The concentrations associated with the release were sampled at a height of $1.5 \mathrm{~m}$ along several cross-wind arcs at downwind distances ranging from 50 to $800 \mathrm{~m}$.

These findings suggest that negative exponential and inverse power-laws are not competing candidate forms of the contact distribution but are instead representative of different atmospheric conditions. Inverse power-laws are more prevalent. This in turn suggests that the atmospheric dispersal of fungal spores will tend to give rise to dispersive wave epidemics. This assertion finds support in recent empirical studies. Cowger et al. (9) and Mundt et al. (30), for example, reported that the wind-seasonal spread of wheat stripe rust (caused by Puccinia striiformis on wheat Triticum aestivum) at spatial scales $<100 \mathrm{~m}$ in experimental plots were empirically consistent with dispersive wave dynamics and so with an inverse power-law contact distribution. Historical plant disease epidemics (potato late blight, wheat stem rust, and southern corn leaf blight) at the continental scale are also consistent with inverse power-law contact distributions (30).

It is hoped that this study will motivate a reappraisal of the appropriateness of contact distributions with exponential and inverse power-law tails, and prompt further studies into role that atmospheric conditions may play in instigating travelling wave and dispersive wave epidemics. In this regard it is worth noting that the spatiotemporal of disease epidemic dynamics resulting from the airborne dispersal of pathogenic spores characterized by power-law contact distributions are governed by fractional diffusion equations. Fractional diffusion equations have been studied intensively and have found numerous applications (28) but until now have not featured in the literature on plant disease epidemics. It would be interesting to see whether this understanding can be exploited in a plant disease epidemic setting.

\section{ACKNOWLEDGMENTS}

Rothamsted Research receives grant support from the Biotechnology and Biological Sciences Research Council. I thank F. van den Bosch and J. West for useful comments on plant disease epidemics and for constructive commentaries on earlier versions of the manuscript.

\section{LITERATURE CITED}

1. Austerlitz, F., Dick, C. W., Dutech, C., Klein, E. K., Oddou-Muratorio, S., Smouse, P. E., and Sork, V. L. 2004. Using genetic markers to estimate the pollen dispersal curve. Mol. Ecol. 13:937-945.

2. Aylor, D. E., and Flesch, T. K. 2001. Estimating spore release rates using a Lagrangian stochastic simulation model. J. Appl. Meteorol. 40:1196-1208.

3. Barad, M. L. 1958. Project Prairie Grass, A field program in diffusion. Vol. 1 Geophysics Research Paper No. 59, Air Force Cambridge Research Center, Bedford, Massachusetts.

4. Boehm, M., Aylor, D. E., and Shields, E. J. 2008. Maize pollen dispersal under convective conditions. J. Appl. Meteorol. Climatol. 47:291-307.

5. Bohrer, G., Katul, G. G., Nathan, R., Walko, R. L., and Avissar, R. 2008. Effects of canopy heterogeneity, seed abscission and inertia on winddriven contact distributions of tree seeds. J. Ecol. 96:569-580.

6. Bullock, J. M., and Clarke, R. T. 2000. Long-distance seed dispersal by wind: Measuring and modelling the tail of the curve. Oecologia 124:506521.

7. Cannas, S. A., Marco, D. E., and Montemurro, M. A. 2006. Long range dispersal and spatial pattern formation in biological invasions. Math. Biosci. 203:155-170.

8. Clark, J. S., Lewis, M., and Horvath, L. 2001. Invasion by extremes: Population spread with variation in dispersal and reproduction. Am. Nat. 157:537-554.

9. Cowger, C., Wallace, L. D., and Mundt, C. C. 2005. Velocity of spread of wheat stripe rust epidemics. Phytopathology 95:972-832.
10. Damgaard, C., and Kjellsson, G. 2005. Gene flow of oilseed rape (Brassica napus) according to isolation distance and buffer zone. Agric. Sys. Environ. 108:291-301.

11. Devaux, C., Lavigne, C., Falentin-Guyomarch H., Vautrin, S., Lecomte, J., and Klein, E. K. 2005. High diversity of oilseed rape pollen clouds over an agro-ecosystem indicates long-distance dispersal. Mol. Ecol. 14:2269-2280.

12. Du, S., and Venkatram, A. 1998. The effect of streamwise diffusion on ground-level concentrations. Atmos. Environ. 32:1955-1961.

13. Ferrandino, F. J. 1993. Dispersive epidemic waves. I. Focus expansion within a linear planting. Phytopathology 83:795-802.

14. Finnigan, J. 2000. Turbulence in plant canopies. Annu. Rev. Fluid Mech. 32:519-571.

15. Fitt, B. D. L., Gregory, P. H., Todd, A. D., McCartney, H. A., and MacDonald, O. C. 1987. Spore dispersal and plant disease gradients; A comparison between two empirical models. J. Phytopathol. 118:227-242.

16. Flesch, T. K., and Wilson, J. D. 1992. A two-dimensional trajectorysimulation model for non-Gaussian, inhomogeneous turbulence within plant canopies. Boundary-Layer Meteorol. 61:349:374

17. Gnedenko, B. V., and Kolmogorov, A. N. 1954. Limit Distributions for Sums of Independent Random Variables. Addison-Wesley, Cambridge, MA.

18. Gryning, S. E., and Lyck, E. 1984. Atmospheric dispersion from elevated sources in an urban area: Comparisons between tracer experiments and model calculations. J. Appl. Meteorol. Climatol. 23:651-660.

19. Hastings, A., Cuddington, K., Davis, K. F., Dugaw, C. J., Elmendorf, S., Freestone, A., Harrison, S., Holland, M., Lambrinos, J., Malvadkar, U., Melbourne, B. A., Moore, K., Taylor, C., and Thomson, D. 2005. The spatial spread of invasions: New developments in theory and evidence. Ecol. Lett. 8:91-101

20. Jarosz, N., Loubet, B., and Huber, L. 2004. Modeling airborne concentration and deposition rate of maize pollen. Atmos. Environ. 38:55555566.

21. Katul, G. G., Porporato, A., Nathan, R., Siqueira, M., Soons, M. B., Poggi, D., Horn, H. S., and Levin, S. A. 2005. Mechanistic Analytical Models for long-distance seed dispersal by wind. Am. Nat. 166:368-381.

22. Kot, M., Lewis, M. A., and van den Driessche, P. 1996. Dispersal data and the spread of invading organisms. Ecology 77:2027-2042.

23. Kuparinen, A. 2006. Mechanistic models for wind dispersal. Trends Plant Sci. 11:296-301.

24. Lavigne, C., Klein, E. K., Valleé, P., Pierre, J., Godelle, B., and Renard, M. 1998. A pollen-dispersal experiment with transgenic oilseed rape. Estimation of the average pollen dispersal of an individual plant within a field. Theor. Appl. Gen. 96:886-896.

25. Lett, C., and Østergård, H. 2000. A stochastic model simulating the spatiotemporal dynamics of yellow rust on wheat. Acta Phytopathol. Entomol. Hung. 35:287-293.

26. Luhar, A. K., and Britter, R. E. 1998. A random walk model for dispersion in inhomogeneous turbulence in a convective boundary layer. Atmos. Environ. 23:1911-1924.

27. Mason, P. J. 1992. Large-eddy simulation of dispersion in convective boundary layers with wind shear. Atmos. Environ. 26A:1561-1571.

28. Metzler, R., and Klafter, J. 2004. The restaurant at the end of the random walk: Recent developments in the description of anomalous transport by fractional dynamics. J. Physics A 37:R161-R208.

29. Mollison, D. 1977 Spatial contact models for ecological and epidemic spread. J. Roy. Stat. Soc. B 39:283-326.

30. Mundt, C. C., Sackett, K. E., Wallace, L. D., Cowger, C., and Dudley, J. P. 2009. Long-distance dispersal and accelerating waves of disease: Empirical relationships. Am. Nat. 173:456-466.

31. Nathan, R., Katul. G. G., Horn, H. S., Thomas, S. M., Oren, R., Avissar, R., Pacala, S. W., and Levin, S. A. 2002. Mechanisms of long-distance dispersal of seeds by wind. Nature 418:409-413.

32. Reynolds, A. M., Bohan, D. A., and Bell, J. R. 2007. Ballooning dispersal in arthropod taxa: Conditions at take-off. Biol. Lett. 3:237-240.

33. Rodean, H. C. 1996. Stochastic Lagrangian Models of Turbulent Diffusion. American Meteorological Society Monograph, Boston, MA.

34. Rotach, M. W., Gryning, S.-E., and Tassone, C. 1996. A two-dimensional Lagrangian stochastic dispersion model for daytime conditions. Q. J. Roy. Meteorol. Soc. 122:367-389.

35. Shaw, M. W. 1995. Simulation of population expansion and spatial pattern when individual dispersal distributions do not decline exponentially with distance. Proc. Roy. Soc. B 259:243-248.

36. Shaw, M. W., Harwood, T. D., Wilkinson, M. J., and Elliott, L. 2006 Assembling spatially explicit landscape models of pollen and spore dispersal by wind for risk assessment. Proc. Roy. Soc. B 273:1705-1713.

37. Sparre Andersen, E. 1953. On the fluctuations of sums of random variables. Math. Scand. 1:263-285.

38. Sparre Andersen, E. 1954. On the fluctuations of sums of random variables II. Math. Scand. 2:195-223. 
39. Stockmarr, A., Andreasen, V., and Østergård, H. 2007 Dispersal distances for airborne spores based on deposition rates and stochastic modeling. Phytopathology 97:1325-1330.

40. Tennekes, H., and Lumley, J. L. 1972. A First Course in Turbulence. MIT Press, Boston, MA.

41. Thomson, D. J. 1987. Criteria for the selection of stochastic models of particle trajectories in turbulent flows. J. Fluid Mech. 180:529-556.

42. Thomson, D. J., and Montgomery, M. R. 1994. Reflection boundary conditions for random walk models of dispersion in non-Gaussian turbulence. Atmos. Environ. 28:1981-1987.

43. Timmons, A. M., Charters, Y. M., Crawford, J. W., Burn, D., Scott, S. E., Dubbels, S. J., Wilson, N. J., Robertson, A., O'Brien, E. T., Squire, G. R., and Wilkinson, M. J. 1996. Risk from transgenic crops. Nature 380:487487.

44. Veneziani, M., Griffa, A., Reynolds, A. M., and Mariano, A. J. 2004. Oceanic turbulence and stochastic models from subsurface Lagrangian data for the northwest Atlantic Ocean. J. Phys. Oceanog. 34:1884-1906.

45. Venkatram, A., and Du, S. 1997. An analysis of the asymptotic behaviour of cross-wind-integrated ground-level concentrations using Lagrangian stochastic simulation. Atmos. Environ. 31:1467-1476.
46. Weil, J. C., Patton, E. G., and Sullivan, P. P. 2006. Lagrangian modeling of dispersion in the stable boundary-layer. In: Proceedings of the 17th Symposium on Boundary-Layers and Turbulence. American Meteorological Society, Boston, MA.

47. White, E. P., Enquist, B. J., and Green, J. L. 2008. On estimating the exponent of power-law frequency distributions. Ecology 89:905-912.

48. Willis, G. E., and Deardorff, J. W. 1976. A laboratory model of diffusion into the convective planetary boundary-layer. Q. J. Roy. Meteorol. Soc. 102:427-445.

49. Willis, G. E., and Deardorff, J. W. 1978. A laboratory study of dispersion from an elevated source within a modeled convective mixed layer. Atmos. Environ. 12:1305-1311.

50. Willis, G. E., and Deardorff, J. W. 1981. A laboratory study of dispersion from a source in the middle of a convective mixed layer. Atmos. Environ. 15:109-117.

51. Wingen, L. U., Brown, J. K. M., and Shaw, M. W. 2007. The population genetic structure of clonal organisms generated by exponentially bounded and fat-tailed dispersal. Genetics 177:435-448.

52. Zadoks, J. C. 2001. Plant disease epidemiology in the twentieth century: A picture by means of selected controversies. Plant Dis. 85:808-816. 